\title{
G-CSF and IL-8 but not GM-CSF correlate with severity of pulmonary neutrophilia in acute respiratory distress syndrome
}

\author{
A. Aggarwal*, C.S. Baker*, T.W. Evans**, P.L. Haslam*
}

\begin{abstract}
G-CSF and IL-8 but not GM-CSF correlate with severity of pulmonary neutrophilia in acute respiratory distress syndrome. A. Aggarwal, C.S. Baker, T.W. Evans, P.L. Haslam. (C) ERS Journals Ltd 2000.

ABSTRACT: Activated neutrophils play a major role in the pathogenesis of acute respiratory distress syndrome (ARDS), and persistence of pulmonary neutrophilia is related to poor survival. Interleukin (IL)-8 is implicated in recruiting neutrophils to the lungs but it has been postulated that granulocyte-macrophage colony-stimulating factor (GM-CSF) and granulocyte colony-stimulating factor (G-CSF), which can promote the survival of neutrophils by delaying apoptosis, may prolong the inflammatory response. The aim of this study was to investigate the levels of GM-CSF and G-CSF in the lungs of patients with ARDS and determine their relationship relative to IL-8 with levels of neutrophils and clinical outcome.

The lungs of 31 patients with ARDS were sampled by means of bronchoalveolar lavage (BAL) and assays of the three cytokines were conducted via enzyme-linked immunosorbent assay.
\end{abstract}

GM-CSF, G-CSF and IL-8 were all increased in the patients compared to healthy controls but concentrations of GM-CSF were much lower than those of G-CSF and IL-8 (GM-CSF $<$ G-CSF $<$ IL-8). Levels of G-CSF and IL-8, but not GM-CSF, correlated strongly with each other $(\mathrm{rS}=0.86, \mathrm{p}<0.001)$ and with $\mathrm{BAL}$ neutrophil counts, and only levels of G-CSF were significantly higher in nonsurvivors than survivors $(\mathbf{p}<\mathbf{0 . 0 5})$.

This evidence indicates that granulocyte colony-stimulating factor as well as interleukin-8 plays a role in the mechanisms of pulmonary neutrophilia in acute respiratory distress syndrome, whereas the role of granulocyte-macrophage colony-stimulating factor remains unclear. The higher levels of granulocyte colony-stimulating factor in nonsurvivors, together with previous reports that recombinant granulocyte colonystimulating factor and granulocyte-macrophage colony-stimulating factor occasionally induce acute lung injury, emphasize that the role of these mediators in pathogenesis needs to be elucidated.

Eur Respir J 2000; 15: 895-901.

\author{
* Cell Biology Unit, National Heart and \\ Lung Institute and ** Unit of Critical Care, \\ Royal Brompton Campus, Imperial College \\ School of Medicine, London, UK. \\ Correspondence: P.L Haslam \\ Cell Biology Unit \\ National Heart and Lung Institute \\ Imperial College School of Medicine \\ Dovehouse Street \\ London SW3 6LY \\ UK \\ Fax: 442073763442 \\ Keywords: Acute respiratory distress \\ syndrome \\ granulocyte colony-stimulating factor \\ granulocyte macrophage colony- \\ stimulating factor \\ interleukin-8 \\ neutrophils \\ prognosis
}

Received: December 91999

Accepted after revision January 282000

This study was supported by the British Lung Foundation.
Acute respiratory distress syndrome (ARDS) can develop in many serious conditions including sepsis, pneumonia, traumatic injury and major surgery $[1,2]$. Mortality is $>40 \%$ and there is no effective therapy apart from supportive measures [2]. The initiating factors trigger an acute systemic inflammatory response which causes microvascular damage leading to increased pulmonary vascular permeability, interstitial and alveolar oedema, and hypoxaemic respiratory failure $[1,2]$. Activated neutrophils play a major role in mediating the microvascular damage and also contribute to lung tissue damage [3]. They infiltrate the lungs in large numbers and their persistence in the lungs is an important determinant of poor survival [4, 5]. Elucidation of the mechanisms that maintain pulmonary neutrophilia in ARDS may therefore be of considerable prognostic and therapeutic significance.

The main chemotactic factor for neutrophils in the blood and bronchoalveolar lavage (BAL) fluids of patients with ARDS has been shown to be the cytokine interleukin (IL)8 , and other products of inflammation make a lesser contribution [6]. Higher levels of IL-8 are present in BAL samples from nonsurvivors than survivors with ARDS [7], and elevations of IL-8 concentration in BAL fluid predict progression to ARDS in at-risk populations [8]. Assays of chemotactic function indicate that IL-8 accounts for $>50 \%$ of the neutrophil chemotactic activity in BAL fluids of patients with ARDS [9].

However, evidence is emerging that factors that have the capacity to prolong neutrophil survival may also be present in the lungs of patients with ARDS. Circulating neutrophils have a short life span of 6-8 h due to programmed cell death, "apoptosis", which normally ensures that the effete cells are recognized and cleared by phagocytes and other cells without spilling their potentially toxic contents [10]. Recent morphological evidence suggests that apoptosis of neutrophils is suppressed during the course of ARDS [11]. Furthermore, BAL fluid from the same patients prolonged the life span of normal neutrophils in vitro. This effect was prevented by preincubation of the fluids with antibodies directed against the cytokines granulocyte-macrophage colony-stimulating factor (GMCSF) and granulocyte colony-stimulating factor (G-CSF). 
In addition to their ability to promote increased release of granulocytes from the bone marrow, both of these cytokines can prolong the life span of neutrophils in vitro by delaying their apoptosis [12]. However, little is known about in vivo levels of GM-CSF or G-CSF in the lungs of patients with ARDS, or their potential role in the pathogenesis of this syndrome. The main aims of this study, therefore, were to: 1) evaluate levels of GM-CSF and G-CSF in the lungs of patients with ARDS at different stages in the injury by studying samples of alveolar epithelial lining fluid obtained by means of BAL; 2) determine their relationship to numbers of BAL neutrophils and to IL-8; and 3) investigate these mediators in regard to clinical outcome.

\section{Methods}

\section{Study groups}

Thirty-one patients with ARDS (19 male, 12 female; median age $37 \mathrm{yrs}$, range 18-66 yrs, 14 never, nine current and eight exsmokers; 22 survivors and nine nonsurvivors) who met the diagnostic criteria of the American/European Consensus [1], and had severe lung injury according to the Murray lung injury score [2] were investigated. The onset of injury was associated with bacterial or viral pneumonia in 10 patients (four of whom also had sepsis), sepsis alone in three, cardiopulmonary bypass surgery in six (one of whom also had sepsis), other major surgery in four (one of whom also had sepsis), major trauma due to road traffic accidents in five, pancreatitis in one, aspiration pneumonia in one and smoke inhalation in one. All had bilateral diffuse parenchymal shadows on their chest radiographs, had developed acute-onset severe hypoxaemia shortly after encountering the risk factor and had an arterial oxygen tension $\left(\mathrm{Pa}_{\mathrm{a}} \mathrm{O}_{2}\right)$ (in $\left.\mathrm{kPa}\right)$ /inspiratory oxygen fraction $\left(F \mathrm{I}_{2} \mathrm{O}_{2}\right)$ ratio of $\leq 21.6$ at some stage during the injury. All had a pulmonary artery wedge pressure of $<18 \mathrm{mmHg}$. Table 1 shows the clinical details. The study protocol was approved by the Ethics Committee of the Royal Brompton Hospital Trust. The control group for the cytokine studies comprised seven healthy nonsmoking volunteers without evidence of lung or heart disease (median age 34 yrs, range 29-42 yrs).

\section{Bronchoalveolar lavage and study protocol}

BAL was performed via a fibreoptic bronchoscope wedged into the lateral segment of the lower lobe of the right lung. The bronchoscope was inserted through the endotracheal tube in the patients and transnasally under local anaesthetic in the healthy volunteers The standard protocol at the authors' centre accords with European guidelines [13]. Briefly, a standard volume of four aliquots of $60 \mathrm{~mL}$ normal saline, buffered to $\mathrm{pH} 7.0$ using $8.4 \%$ sodium bicarbonate, was sequentially introduced and aspirated into a sterile siliconized glass bottle and immediately transferred on ice to the laboratory. The total standard input was achieved in the controls, but in the severely ill ARDS patients instillation was aborted if falls in oxygenation caused clinical concern; thus the introduction volumes were lower in most cases (table 1). The proportions of the fluid recovered were also significantly lower in the patient group compared to the healthy con- trols (table 1), presumably due to the substantial increase in lung permeability that is characteristic of this syndrome $[1,2]$. The BAL fluid was centrifuged at $160 \times g$ for 10 min at $4{ }^{\circ} \mathrm{C}$ to sediment the cells, and the supernatant divided into aliquots, frozen and stored at $-70^{\circ} \mathrm{C}$ in the presence of $1.8 \mathrm{mg} \cdot \mathrm{mL}^{-1}$ dipotassium ethylene diamine tetra acetic acid (EDTA). It was planned to obtain a BAL sample from each patient during the first week on ventilation and again at weekly intervals until successfully weaned in survivors or until death. However, the high mortality rate and other clinical constraints prevented sampling every patient at all follow-up intervals. In total, 15 patients were sampled during the first week "earlier phase" of injury (nine survivors and six nonsurvivors), 12 in the second or third week "mid phase" (10 survivors and two nonsurvivors) and eight survivors during the "later stages" in weeks 4-9 (seven survivors and one nonsurvivor). During the first week, enough samples were available to undertake statistical analysis to determine whether any differences could be identified between the survivors and nonsurvivors. The prognostic value of the

Table 1. - Clinical characteristics of the acute respiratory distress syndrome (ARDS) patients

\begin{tabular}{|c|c|c|}
\hline & Patients & Controls \\
\hline Patients $\mathrm{n}$ & 31 & 21 \\
\hline Sex male/female & $19 / 12$ & \\
\hline Smoking current/ex-never & 9/7/13 & \\
\hline $\begin{array}{l}\text { Outcome survivors/ } \\
\text { nonsurvivors }\end{array}$ & $22 / 9$ & \\
\hline $\begin{array}{l}\text { Onset of respiratory } \\
\text { failure }\end{array}$ & Acute & \\
\hline Chest radiograph & $\begin{array}{l}\text { Bilateral } \\
\text { infiltrates }\end{array}$ & \\
\hline $\mathrm{PAOP}^{+} \mathrm{mmHg}$ & $<18$ & \\
\hline Age yrs & $37(18-66)$ & \\
\hline Minimum $P \mathrm{a}, \mathrm{O}_{2} / F \mathrm{I}, \mathrm{O}_{2}{ }^{\#}$ & $9.38(3.3-20)$ & \\
\hline $\begin{array}{l}{\mathrm{Pa}, \mathrm{O}_{2} / F \mathrm{I}, \mathrm{O}_{2} \text { at time }} \\
\text { of initial } \mathrm{BAL}\end{array}$ & $22.5(9.13-52.2)$ & \\
\hline Total time on MV days & $35(7-130)$ & \\
\hline $\begin{array}{l}\text { Time on MV at } \\
\text { initial BAL days }\end{array}$ & $8(3-59)$ & \\
\hline \multicolumn{3}{|l|}{ Initial BAL results: } \\
\hline BAL input volume $\mathrm{mL}$ & $180(40-240)^{* * *}$ & $240(120-240)$ \\
\hline BAL output volume $\mathrm{mL}$ & $38(10-80)^{* * *}$ & $130(75-200)$ \\
\hline BAL fluid recovery $\%$ & $29(6-68)^{* * *}$ & $63(31-85)$ \\
\hline \multicolumn{3}{|l|}{ BAL cell counts: } \\
\hline Neutrophils \% & $\begin{array}{c}78.49 \\
(14.01-98.40)^{* * *}\end{array}$ & $\begin{array}{c}0.70 \\
(0-3.27)\end{array}$ \\
\hline Neutrophils & 14.71 & 0.05 \\
\hline$\times 10^{4}$ cells $\cdot \mathrm{mL}^{-1}$ & $(0.82-203.40)^{* * *}$ & $(0-0.19)$ \\
\hline Macrophages \% & $\begin{array}{c}18.81 \\
(0-74.69)^{* * *}\end{array}$ & $\begin{array}{c}79.50 \\
(55.70-95.84)\end{array}$ \\
\hline $\begin{array}{l}\text { Macrophages } \\
\times 10^{4} \text { cells } \cdot \mathrm{mL}^{-1}\end{array}$ & $\begin{array}{c}2.96 \\
(0-256.00)\end{array}$ & $\begin{array}{c}7.25 \\
(1.28-45.09)\end{array}$ \\
\hline
\end{tabular}

Data are presented as absolute values or median (range). ${ }^{+}$: pulmonary artery occlusion pressure (PAOP) of $<18 \mathrm{mmHg}$ conforms with the diagnostic criteria for ARDS $[1,2] ;{ }^{\#}$ : patient's lowest value for arterial oxygen tension $\left(\mathrm{Pa}_{\mathrm{a}} \mathrm{O}_{2} ;\right.$ in $\left.\mathrm{kPa}\right) /$ inspiratory oxygen fraction $\left(F \mathrm{I}, \mathrm{O}_{2}\right)$. ARDS is defined by a $P \mathrm{a}, \mathrm{O}_{2} / F \mathrm{I}, \mathrm{O}_{2}$ of $\leq 21.6$ according to MurRAY et al. [2] or $\leq 26.7$ according to BERNARD et al. [1]. All patients fulfilled one of these criteria at some time during their illness. BAL: bronchoalveolar lavage; MV: mechanical ventilation. ${ }^{* * *}: \mathrm{p}<0.001$ compared to healthy controls (Mann-Whitney U-test). 
initial BAL findings for all 31 patients was also investigated. Survival was defined as successful weaning from ventilation and discharge from intensive care. A single BAL sample was obtained from each of the controls.

\section{Bronchoalveolar lavage cell counts}

Total counts of nucleated cells were carried out using Kimura stain and an improved Neubauer counting chamber (Hawksley and Sons Ltd., London, UK). Results were expressed as the numbers of cells per millilitre of BAL fluid. Differential counts to identify the cells were performed by making cytocentrifuge slide preparations containing $1.5 \times 10^{5}$ cells stained with May-Grünwald-Giemsa. At least 300 cells were counted in random fields and the results expressed for each cell type as a percentage of the total cell population.

Granulocyte colony-stimulating factor, granulocyte-macrophage colony-stimulating factor and interleukin-8 assays

Levels were determined in BAL fluid supernatants containing EDTA using sandwich enzyme-linked immunosorbent assay kits (R\&D Systems, Abingdon, UK). All standards and samples were assayed in duplicate and concentrations were determined by extrapolation from standard curves. G-CSF was determined using the Quantikine ${ }^{\mathrm{TM}}$ human G-CSF immunoassay kit (Catalogue No. DCS50), validated against international reference preparation National Institute for Biological Standards and Control (NIBSC) 88/ 502. GM-CSF was measured using the high-sensitivity Quantikine ${ }^{\mathrm{TM}}$ HS human GM-CSF immunoassay kit (Catalogue No. HSGM0). IL-8 was evaluated using the standard Quantikine $^{\mathrm{TM}}$ human IL-8 immunoassay kit (Catalogue No. D8050), validated against international reference preparation NIBSC/World Health Organization 89/520.

\section{Statistical analysis}

Data distributions were nonparametric; thus comparisons between independent groups were made using the Mann-Whitney U-test. Correlations were carried out using the Spearman rank correlation coefficient.
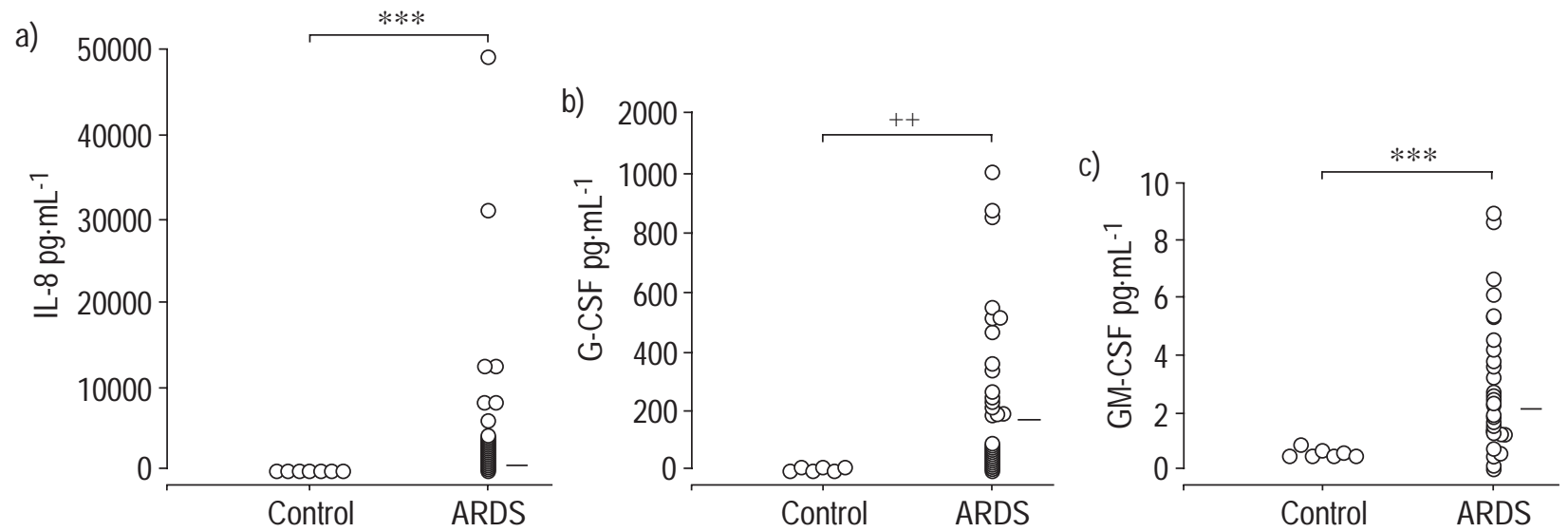
levels

The clinical characteristics of the 31 patients with ARDS and their BAL cell counts at the time of initial lavage are shown in table 1 . All the patients showed increased percentages of neutrophils and highly significant increases in neutrophil numbers per millilitre of BAL fluid recovered compared to healthy controls $(p<0.001)$. The results of cytokine analysis in the same initial lavage samples are shown in figure 1 . These confirm that patients with ARDS have highly significant increases in IL-8 level compared to controls (median (range) 1,350 (1.00-49, 631) versus $\left.9.00 \mathrm{pg} \cdot \mathrm{mL}^{-1}(0-24.50) \mathrm{p}<0.001\right)$, and also show that these patients exhibit highly significant increases in G-CSF $\left(175(0-1,000)\right.$ versus $4.55 \mathrm{pg} \cdot \mathrm{mL}^{-1}(0-$ $6.50) \mathrm{p}<0.0025)$ and GM-CSF levels $(2.20(0-8.90)$ versus $\left.0.39 \mathrm{pg} \cdot \mathrm{mL}^{-1}(0.32-0.71) \mathrm{p}<0.001\right)$. There was no significant difference in the cytokine levels or neutrophil counts between the 15 patients with infection (primary or secondary) and the 16 without infection. However, GCSF levels were significantly higher in the nine patients who failed to survive than in the 22 survivors $(p<0.05$; table 2). Levels of IL-8 and neutrophils in the same samples also tended to be higher in the nonsurvivors. There was no relationship between GM-CSF levels and survival.

\section{Correlation between levels of cytokines and neutrophil counts}

The correlation between numbers of neutrophils and concentrations of each cytokine in the same BAL samples from the patients is shown in table 3 . There were significant correlations between both concentrations of IL-8 and G-CSF and neutrophil percentages and neutrophil numbers per millilitre. By contrast, there was no correlation between concentrations of GM-CSF and neutrophil counts. Consistent with these findings, IL-8 and G-CSF levels correlated closely with each other $(\mathrm{rS}=0.86, \mathrm{p}<$ $0.001)$ but less closely with GM-CSF concentrations in the same lavage samples ( $\mathrm{rs}=0.449, \mathrm{p}<0.025$ and $\mathrm{rS}=$ $0.394, \mathrm{p}<0.05$, respectively).

Fig. 1. - Levels of: a) interleukin-8 (IL-8); b) granulocyte colony-stimulating factor (G-CSF); and c) granulocyte-macrophage colony-stimulating factor $(\mathrm{GM}-\mathrm{CSF})$ in the patients with acute respiratory distress syndrome (ARDS; $n=31)$ at initial lavage compared with healthy control subjects $(\mathrm{n}=7$ ( $\mathrm{n}=6$ for G-CSF)). ${ }^{++}: \mathrm{p}<0.0025 ; * * *: \mathrm{p}<0.001$. 
Table 2. - Bronchoalveolar lavage (BAL) findings in nonsurvivors compared with survivors in the acute respiratory distress syndrome group at initial lavage

\begin{tabular}{lccc}
\hline BAL marker & Nonsurvivors & Survivors & p-value \\
\hline Patients n & 9 & 22 & \\
Neutrophils \% & 91.27 & 43.72 & NS \\
& $(32.85-94.90)$ & $(14.01-98.40)$ & \\
Neutrophils & 24.96 & 10.86 & NS \\
$\times 10^{4} \mathrm{cells} \cdot \mathrm{mL}^{-1}$ & $(4.93-203.45)$ & $(0.82-141.30)$ & \\
IL-8 pg $\cdot \mathrm{mL}^{-1}$ & 2617.60 & 1170.00 & NS \\
& $(133-12352)$ & $(1-49631)$ & \\
G-CSF pg.mL $\mathrm{mL}^{-1}$ & 457 & 80 & $<0.05$ \\
& $(4.34-1000)$ & $(0-866)$ & \\
GM-CSF pg $\cdot \mathrm{mL}^{-1}$ & 2.63 & 1.87 & NS \\
& $(0.49-8.90)$ & $(0-8.60)$ & \\
\hline
\end{tabular}

Data are presented as median (range). IL-8: interleukin-8; GCSF: granulocyte colony-stimulating factor; GM-CSF: granulocyte-macrophage colony-stimulating factor.

\section{Influence of stage in injury on the findings}

Due to clinical constraints, the initial lavage samples from the 31 patients were not all obtained during the first week of injury; therefore, a further analysis was undertaken to better define the influence of time of sampling on the findings. Fifteen patients were lavaged during their first week on mechanical ventilation, 12 during weeks 2 or 3 and eight patients during weeks 4-9, while still receiving ventilatory support. The results are shown in figures 2 and 3. Compared to controls, there were significant increases in neutrophil counts and IL-8 and GM-CSF concentrations at all three time phases, although the levels tended to be lower during the last phase. There were also significant increases in G-CSF concentrations during the first and second time phases but, unlike the results for the other markers, levels of this cytokine were not significantly increased during the last phase, by which time most of the patients remaining were survivors (seven survivors, one nonsurvivor). During the first week, samples were available from nine survivors and six nonsurvivors. At this earlier stage, trends of prognostic differences were identified in those who did not survive towards higher concentrations of G-CSF (nonsurvivors median (range) $48 \mathrm{pg} \cdot \mathrm{mL}^{-1}$ (42-1,000); survivors $\left.174 \mathrm{pg} \cdot \mathrm{mL}^{-1}(10.55-866) \mathrm{p}=0.08\right)$ and neutrophil counts (nonsurvivors median (range) 32.48 $\times 10^{4}$ cell $\cdot \mathrm{mL}^{-1}(18.32-203.45)$; survivors $18.57 \times 10^{4}$ cell. $\left.\mathrm{mL}^{-1}(1.43-85.91) \mathrm{p}=0.09\right)$.

Table 3. - Correlation between levels of cytokines and neutrophils in the 31 patients at initial lavage

\begin{tabular}{|c|c|c|c|c|}
\hline & \multicolumn{2}{|c|}{$\begin{array}{c}\text { Neutrophils } \\
\%\end{array}$} & \multicolumn{2}{|c|}{$\begin{array}{l}\text { Neutrophils } \\
\times 10^{4} \text { cells } \cdot \mathrm{mL}^{-1}\end{array}$} \\
\hline & rs & p-value & rS & p-value \\
\hline $\mathrm{IL}-8 \mathrm{pg} \cdot \mathrm{mL}^{-1}$ & 0.62 & $<0.001$ & 0.43 & $<0.025$ \\
\hline $\mathrm{G}-\mathrm{CSF} \mathrm{pg} \cdot \mathrm{mL}^{-1}$ & 0.54 & $<0.0025$ & 0.42 & $<0.025$ \\
\hline GM-CSF pg.mL ${ }^{-1}$ & 0.22 & NS & 0.23 & NS \\
\hline
\end{tabular}

IL-8: interleukin-8; G-CSF: granulocyte colony-stimulating factor; GM-CSF: granulocyte-macrophage colony-stimulating factor.
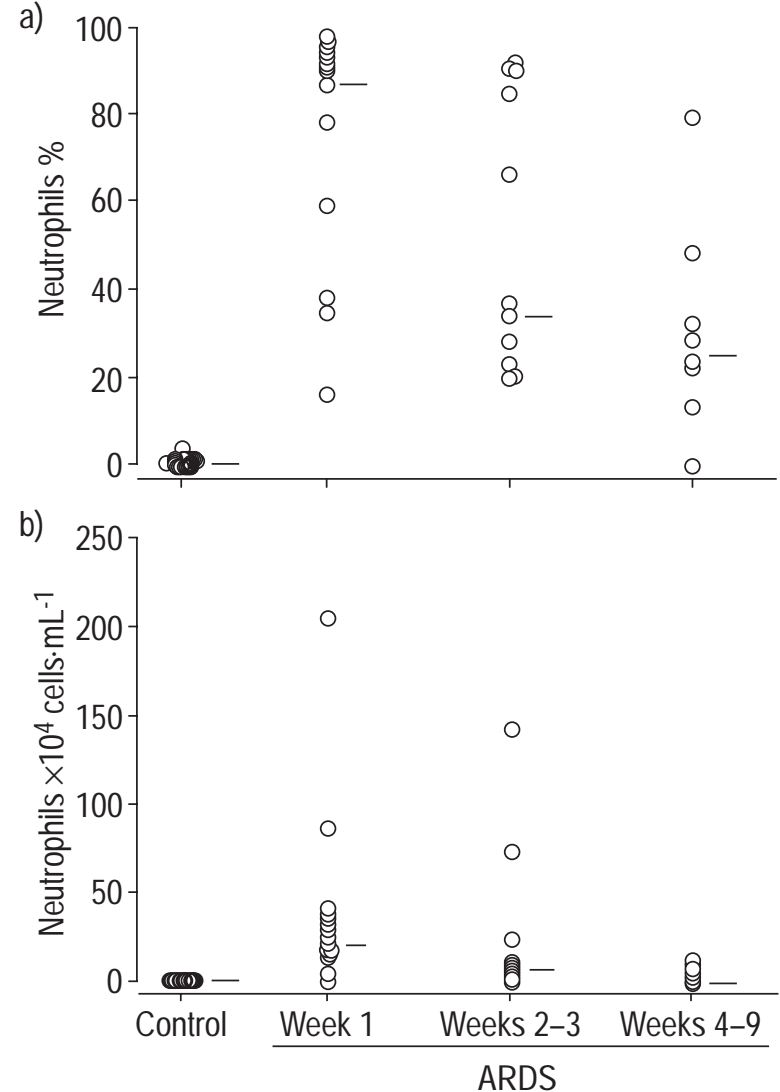

Fig. 2. - Neutrophil counts in bronchoalveolar lavage samples from patients with acute respiratory distress syndrome (ARDS) during the first week of injury $(\mathrm{n}=15)$ compared with those during weeks 2-3 $(n=12)$ and weeks 4-9 $(n=8)$ after onset. Counts for healthy controls $(n=21)$ are also shown. Horizontal bars indicate medians. Patient samples were significantly different from controls $(\mathrm{p}<0.001)$ at all time points and for both parameters.

\section{Discussion}

This study confirms previous reports that IL-8, a potent neutrophil chemokine, is elevated in BAL fluid from patients with ARDS and correlates with the numbers of neutrophils present, indicating that it plays a major role in promoting the massive pulmonary neutrophilia that mediates lung damage [6-9]. It also confirms that neutrophil counts and IL-8 levels tend to be higher in nonsurvivors $[5,7,14,15]$. However, this study shows that these patients also have increased concentrations of G-CSF in BAL, which correlate closely with the levels of neutrophils and IL-8. Moreover, the levels of G-CSF were a stronger predictor of poor outcome than that of either neutrophils or IL-8. This provides the first direct in vivo evidence in patients with ARDS that this factor, which has the ability to prolong the survival of neutrophils by delaying apoptosis [12], may also play a role in the pathogenesis of ARDS.

There has been one previous study of G-CSF levels in BAL fluid from patients with ARDS [11]. They were on average 10-fold higher than those in BAL from normal volunteers (median (range) $238 \mathrm{pg} \cdot \mathrm{mL}^{-1}(19.5-5,241)$ versus mean \pm SEM $25 \pm 20 \mathrm{pg} \cdot \mathrm{mL}^{-1}$ ), but were measured only on the first day after onset of ARDS, and the 


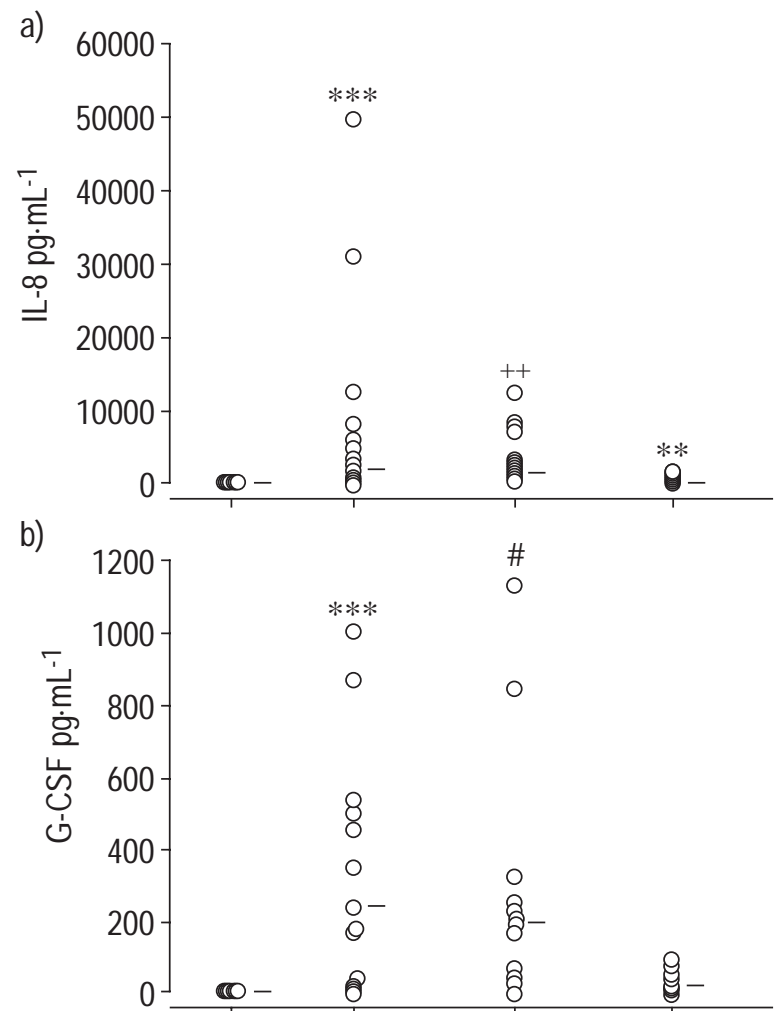

c)

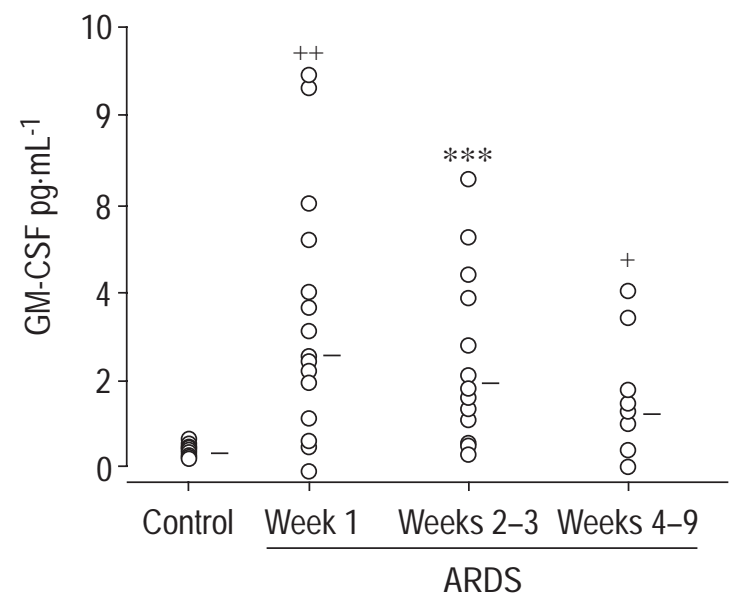

Fig. 3. - Levels of: a) interleukin-8 (IL-8); b) granulocyte colonystimulating factor (G-CSF); and c) granulocyte-macrophage colonystimulating factor (GM-CSF) in bronchoalveolar lavage samples from patients with acute respiratory distress syndrome (ARDS) during the first week of injury $(n=15)$ compared with those during weeks $2-3$ $(\mathrm{n}=12)$ and weeks 4-9 $(\mathrm{n}=8)$ after onset. ${ }^{+}: \mathrm{p}<0.025 ;^{++}: \mathrm{p}<0.0025 ; * *$ : $\mathrm{p}<0.01$; $^{\#}: \mathrm{p}<0.005 ; * * *: \mathrm{p}<0.001$ compared to control subjects.

relationship with neutrophil counts and clinical outcome was not investigated. The main aim of the study was to seek evidence that apoptosis of neutrophils might be inhibited in the lungs of patients with ARDS. In support of this hypothesis, the authors showed that the proportion of apoptotic neutrophils in BAL fluid was low (median $\leq 3 \%$ ) throughout the course of ARDS (days 1, 3, 7, 14 and 21), and similarly low in patients at risk. These findings alone are difficult to interpret because apoptotic cells are rarely detected in vivo due to their very rapid clearance by phagocytes [16]. More importantly, BAL fluid from their patients with ARDS reduced the rate of apoptosis of blood neutrophils in vitro [11]. Furthermore, the anti-apoptotic effect could be blocked by preincubating BAL fluids with polyclonal antibodies directed against G-CSF and GM-CSF, indicating that both cytokines may prolong the survival of neutrophils in the lungs of patients with ARDS. The present results extend these findings by documenting the levels of both these cytokines in vivo in the lungs of patients with ARDS at different stages in the progression of the syndrome, and by defining their relationship with the neutrophilic response and patient outcome.

The evidence presented here also supports a role for GCSF in vivo in the mechanisms that promote the severe pulmonary neutrophilia that is associated with poor survival in ARDS $[4,5]$. The concentrations of G-CSF in BAL fluid during the first week of injury (median (range) 240 $\left.\mathrm{pg} \cdot \mathrm{mL}^{-1}(11-1,000)\right)$ are similar to those reported in the previous study on the first day after the onset of injury [11]. Initial levels for the total group of patients were significantly higher in nonsurvivors than survivors $(\mathrm{p}<0.05)$. At later stages in the injury, the levels were lower and were within the normal range in nearly all patients by weeks 4-9. At this stage most of the patients were survivors who were subsequently weaned from ventilation, indicating that levels of G-CSF return to normal with recovery. Counts of neutrophils and levels of IL-8 were also lower during the recovery phase.

By contrast, no significant relationship between concentrations of GM-CSF in BAL fluid and levels of neutrophils, nor prognosis, was found in patients with ARDS in the present study. Its role in the pathogenesis is therefore less clear. The increases in GM-CSF levels in BAL were much lower (median (range) $2.2 \mathrm{pg} \cdot \mathrm{mL}^{-1}(0-8.9)$ than those of G-CSF, and, although in vivo concentrations may be up to 180-fold higher because of the dilution due to $\mathrm{BAL}$, it is not known whether such low concentrations of GM-CSF within the lungs would have any pathophysiological effect. The median tolerated dose of intravenous recombinant GM-CSF is $15 \mu \mathrm{g} \cdot \mathrm{kg}$ body weight ${ }^{-1}$. day $^{-1}$ [17]. The present findings suggest that the numbers of neutrophils in the lungs in ARDS are influenced more by intrapulmonary levels of the mediators G-CSF and IL-8 than by GM-CSF.

Unlike G-CSF, the colony-stimulating and anti-apoptotic effects of GM-CSF are less specific to neutrophils. It pro-longs the survival of eosinophils as well as neutrophils from the blood of normal volunteers in vitro by inhibiting apoptosis [18], promotes the release of monocytes, neutrophils and eosinophils from bone marrow [19] and enhances many of the biological functions of these cells [18]. However, ARDS is a disorder that specifically involves increases only in neutrophil numbers. The absence of increases in numbers of eosinophils and monocytes in ARDS suggests that the low levels of GM-CSF detected in the lungs in vivo may not have a significant colonystimulating effect. The levels were on average five-fold higher than normal, whereas those of G-CSF were 38-fold higher and those of IL-8 150-fold higher. Other functions of GM-CSF may be more relevant to its role in the mechanism of ARDS, which remains to be elucidated.

Experimental studies have provided evidence that G-CSF plays a more important role than GM-CSF in the regulation of blood neutrophil numbers. Dogs depleted of G-CSF by a 
neutralizing antibody develop profound and selective neutropenia [20], but dogs similarly depleted of GM-CSF do not become neutropenic [21]. Furthermore, disruption of the G-CSF gene in knock-out mice results in chronic neutropenia [22], whereas GM-CSF knock-out mice do not become neutropenic [23]. Thus G-CSF appears more important than GM-CSF in the normal regulation of neutrophil homeostasis. There is very little information on levels of G-CSF in the lungs of healthy subjects, but alveolar macrophages recovered by BAL from healthy controls can produce G-CSF after stimulation with endotoxin [24]. The sources of G-CSF in the lungs of patients with ARDS are unknown, but G-CSF can be produced by monocytes, macrophages, endothelial cells and fibroblasts in vitro in response to stimulation by IL-1 and tumour necrosis factor- $\alpha$, which are key inflammatory mediators in ARDS $[25,26]$. In addition, bronchial epithelial cells containing G-CSF messenger ribonucleic acid have been seen in a rat model of acute lung injury [26]. Further studies are now needed to identify the main sources of G-CSF in the lungs of patients with ARDS and to clarify its role in pathogenesis.

The potential of G-CSF to contribute to the pathogenesis of ARDS is supported by reports of development of acute lung injury in patients treated with recombinant G-CSF [28-30]. In addition, experimental models show that intravenous instillation of recombinant G-CSF can exacerbate pre-existing acute lung injury [31-33]. Furthermore, it has recently been reported that G-CSF alone can induce acute lung injury when introduced directly into the lungs of rats [34]. Doses of 300-3,000 ng recombinant human G-CSF introduced intratracheally caused hypoxia, marked pulmonary oedema and pulmonary neutrophilia. The maximum level of G-CSF found in the present study in BAL fluid from patients with ARDS was $2.5 \mathrm{ng} \cdot \mathrm{mL}^{-1}$, but, since the dilution factor due to BAL could reach 180 -fold, in vivo concentrations of G-CSF in lung lining fluid may be up to $450 \mathrm{ng} \cdot \mathrm{mL}^{-1}$, sufficient to have a pathophysiological effect.

In conclusion, this study shows that increases in granulocyte colony-stimulating factor and granulocyte-macrophage colony-stimulation factor levels occur in the lungs of patients with acute respiratory distress syndrome. Their role in the pathogenesis and whether such increases also occur in patients at risk, now needs to be clarified.

\section{References}

1. Bernard GR, Artigas A, Brigham KL, et al. and the Consensus Committee. Report of the American-European Consensus Conference on Acute Respiratory Distress Syndrome: definitions, mechanisms, relevant outcomes, and clinical trial coordination. J Crit Care 1994; 9: 72-81.

2. Murray JF, Matthay MA, Luce JM, Flick MR. An expanded definition of the adult respiratory distress syndrome. Am Rev Respir Dis 1988; 138: 720-723.

3. McNee W, Selby C. Neutrophil traffic in the lungs: role of haemodynamics, cell adhesion and deformability. Thorax 1993, 48: 79-88.

4. Steinberg KP, Milberg JA, Martin TR, Maunder RJ, Cockrill BA, Hudson LD. Evolution of bronchoalveolar cell populations in the adult respiratory distress syndrome. Am J Respir Crit Care Med 1994; 150: 113-122.
5. Baughman RP, Gunther KL, Rashkin MC, Keeton DA, Pattishall EN. Changes in the inflammatory response of the lung during acute respiratory distress syndrome: prognostic indicators. Am J Respir Crit Care Med 1996; 154: 76-81.

6. Kunkel SL, Standiford TJ, Kasahara K, Strieter RM. Interleukin-8 (IL-8): the major neutrophil chemotactic factor in the lung. Exp Lung Res 1991; 17: 17-23.

7. Miller EJ, Cohen AB, Nagao S, et al. Elevated levels of NAP-1/interleukin-8 are present in the airspaces of patients with the adult respiratory distress syndrome and are associated with increased mortality. Am Rev Respir Dis 1992; 146: 427-432.

8. Donnelly SC, Strieter RM, Kunkel SL, et al. Interleukin-8 and development of adult respiratory distress syndrome in at risk patient groups. Lancet 1993; 341: 643-647.

9. Goodman RB, Strieter RM, Martin DP, et al. Inflammatory cytokines in patients with persistence of the acute respiratory distress syndrome. Am J Respir Crit Care Med 1996; 154: 602-611.

10. Haslett C. Granulocyte apoptosis and inflammatory disease. Br Med Bull 1997; 53: 669-683.

11. Matute-Bello G, Liles WC, Radell F II, et al. Neutrophil apoptosis in the acute respiratory distress syndrome. Am Respir Crit Care Med 1997; 156: 1969-1977.

12. Cox G, Gauldie J, Jordana M. Bronchial epithelial cell derived cytokines (G-CSF and GM-CSF) promote the survival of peripheral blood neutrophils in vitro. Am J Respir Cell Mol Biol 1992, 7: 507-513.

13. Haslam PL, Baughman RP, eds. Guidelines for measurement of acellular components and recommendations for standardization of bronchoalveolar lavage (BAL) Report of the European Respiratory Society Task Force. Eur Respir Rev 1999; 9: Review 66.

14. Meduri GU, Kohler G, Tolley E, Stentz F, Postlethwaite A. Inflammatory cytokines in the BAL of patients with ARDS. Persistent elevation over time predicts poor outcome. Clin Invest Crit Care 1995; 108: 1303-1314.

15. Ikuta N, Taniguchi H, Kondoh Y, Takagi K, Hayakawa T. Sustained high levels of circulatory interleukin-8 are associated with a poor outcome in patients with adult respiratory distress syndrome. Intern Med 1996; 35: 855-860.

16. Cox G, Crossley J, Xing Z. Macrophage engulfment of apoptotic neutrophils contributes to the resolution of acute pulmonary inflammation in vivo. Am J Respir Cell Mol Biol 1995; 12: 232-237.

17. Moore MAS. The clinical use of colony stimulating factors. Annu Rev Immunol 1991; 9: 159-191.

18. Lopez AF, Williamson J, Gamble JR, et al. Recombinant granulocyte-macrophage colony-stimulating factor stimulates in vitro mature human neutrophil and eosinophil function, surface receptor expression and survival. $J$ Clin Invest 1986; 78: 1220-1228.

19. Aglietta M, Piacibello W, Sanavio F, et al. Kinetics of human hemopoietic cells after in vivo administration of granulocyte-macrophage colony-stimulating factor. J Clin Invest 1989, 83: 551-557.

20. Hammond WP, Csiba E, Canin A, et al. Chronic neutropenia: a new canine model induced by human G-CSF. $J$ Clin Invest 1991; 87: 704-710.

21. Dranoff G, Crawford AD, Sadelain M, et al. Involvement of granulocyte-macrophage colony-stimulating factor in pulmonary homeostasis. Science 1994; 294: 713-716.

22. Lieschke GJ, Grail D, Hodgson G, et al. Mice lacking granulocyte colony-stimulating factor have chronic neutrophenia, granulocyte and macrophage progenitor cell 
deficiency, and impaired neutrophil mobilization. Blood 1994; 84: 1737-1746.

23. Stanley E, Lieschke GJ, Grail D, et al. Granulocyte-macrophage colony-stimulating factor-deficient mice show no major perturbation of hematopoiesis but develop a characteristic pulmonary pathology. Proc Natl Acad Sci U S A 1994; 91: 5592-5596.

24. Tazi A, Nioche S, Chastre J, Smiejan JM, Hance AJ. Spontaneous release of granulocyte colony-stimulating factor (G-CSF) by alveolar macrophages in the course of bacterial pneumonia and sarcoidosis: endotoxin-dependent and endotoxin-independent G-CSF release by cells recovered by bronchoalveolar lavage. Am J Respir Cell Mol Biol 1991; 4: 140-147.

25. Rambaldi A, Young DC, Griffin JD. Expression of the MCSF (CSF-1) gene by human monocytes. Blood 1987; 69: 1409-1413.

26. Seelentag WK, Mermod JJ, Montesano R, Vassalli P. Additive effects of interleukin-1 and tumor necrosis factor- $\alpha$ on the accumulation of the three granulocyte and macrophage colony stimulating factor mRNAs in human endothelial cells. EMBO J 1987; 6: 2261-2265.

27. Hierholzer C, Kelly E, Tsukada K, et al. Hemorrhagic shock induces granulocyte colony-stimulating factor (GCSF) expression in bronchial epithelium. Am J Physiol 1997; 273: L1058-L1064.
28. Takahashi Y, Kobayashi Y, Chikayama S, Ikeda M, Kondo M. Effect of granulocyte/colony-stimulating factor on the onset of the adult respiratory distress syndrome. Acta Haematol 1999; 101: 124-129.

29. Nelson S. A question of balance. Am J Respir Crit Care Med 1999; 159: 1365-1367.

30. Schilero GJ, Oropello J, Benjamin E. Impairment in gas exchange after granulocyte colony stimulating factor (G$\mathrm{CSF}$ ) in a patient with the adult respiratory distress syndrome. Chest 1995; 107: 276-278.

31. King J, DeBoisblanc BP, Mason CM, et al. Effect of granulocyte colony-stimulating factor on acute lung injury in the rat. Am J Respir Crit Care Med 1995; 151:302-309.

32. Terashima T, Kanazawa M, Sayama K, et al. Granulocyte colony-stimulating factor exacerbates acute lung injury induced by intratracheal endotoxin in guinea pigs. $\mathrm{Am} J$ Respir Crit Care Med 1994; 149: 1295-1303.

33. Lang CH, Bagby GJ, Dobrescu C, Nelson S, Spitzer JJ. Effect of granulocyte colony-stimulating factor on sepsisinduced changes in neutrophil accumulation and organ glucose uptake. J Infect Dis 1992; 166: 336-343.

34. Hierholzer C, Kelly E, Lyons V, et al. G-CSF instillation into rat lungs mediates neutrophil recruitment, pulmonary edema, and hypoxia. J Leukocyte Biol 1998; 63: $169-174$. 